\title{
The Earth's magnetopause as a source and sink for equatorial nightside energetic charged particles
}

\author{
M. M. Klida and T. A. Fritz \\ Center for Space Physics, Boston University, Boston, MA 02215, USA
}

Received: 27 February 2009 - Revised: 30 September 2009 - Accepted: 30 October 2009 - Published: 26 November 2009

\begin{abstract}
The Imaging Proton Spectrometer (IPS) and the Imaging Electron Spectrometer (IES) on the Polar satellite have measured temporary deviations in the isotropy of the pitch angle distributions (PADs) of charged particles in the inner magnetosphere. As Polar passes through the nightside equatorial region, the IPS and IES observe dropouts of charged particles with pitch angles near $90^{\circ}$, known as butterfly distributions caused by the shadowing of the magnetopause. Additionally, Polar observes a lower energy $(<60 \mathrm{keV})$ intensification of locally mirroring ions while simultaneously detecting butterfly PADs in both higher energy ions and electrons. While it is accepted that charged particles can be lost to the magnetopause due to shadowing effects, the modeling here can suggest that the magnetopause can also be a direct source for particles observed in magnetosphere, with a strong dependence upon both pitch angle and particle energy.
\end{abstract}

Keywords. Magnetospheric physics (Energetic particles, trapped; Magnetopause, cusp, and boundary layers; Magnetospheric configuration and dynamics)

\section{Introduction}

The butterfly pitch angle distribution (PAD) was first described by West (1966) with observations of Soviet nuclear testing in the upper atmosphere on 28 October 1962. These PADs are characterized by a decrease in the flux of particles with pitch angles near $90^{\circ}$. This minimum, along with the additional field-aligned minima created by the loss cone, will form a butterfly shaped distribution. Later reports of naturally occurring butterfly PADs in populations of both electrons and protons were made by Serlemitsos (1966), Pfitzer

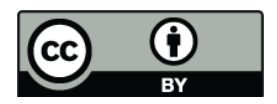

Correspondence to: M. M. Klida (mklida@bu.edu) et al. (1969), Bogott and Mozer (1971), West et al. (1973), West and Buck (1974), Kaye et al. (1978), and Fritz et al. (2003) among others.

Using the Comprehensive Energetic Particle and Pitch Angle Distribution (CEPPAD) instrument package on the Polar satellite (Blake et al., 1995), the characteristics of the PADs of energetic ions and electrons are compared for two specific events. The butterfly shaped PAD, indicating a deficiency of locally mirroring charged particles are commonly observed as Polar passes through the equatorial region of the inner magnetosphere. The orbital dynamics of the satellite have allowed for the observation of a large portion of the radiation belt region. Over a period of about six years, the satellite's apogee precessed from the northern pole to the equator. This corresponds to equatorial crossings from inside $3 R_{E}$ to outside $9 R_{E}$ at all local times. Many mechanisms have been proposed as the cause of the butterfly PAD, and through the modeling of ions and electrons, it can be supported that shadowing by the magnetopause can be at least one of the methods by which both $90^{\circ}$ pitch angle electrons and ions are lost from the equatorial zone (West et al., 1973), and as reported herein, the magnetopause is a source for ions of lower energy $(<60 \mathrm{keV})$ as well.

The compression of the earth's magnetic field due to contact with the solar wind leads to asymmetries in the geometry of the geomagnetic field with a dependence upon magnetic local time. For a given latitude and radial distance, the dayside magnetopause, having been compressed by the dynamic pressure of the solar wind has higher field strengths than the nightside magnetosphere at a given radial distance. This deviation from a perfect magnetic dipole forces charged particles to drift in non-circular drift-paths. For equatorially mirroring particles, the conservation of the first adiabatic invariant will constrain these particles to paths of constant magnetic field strength. Consequently, particles will move radially outward as they drift from the nightside to dayside. Closer toward the earth, electrons and protons can remain

Published by Copernicus Publications on behalf of the European Geosciences Union. 

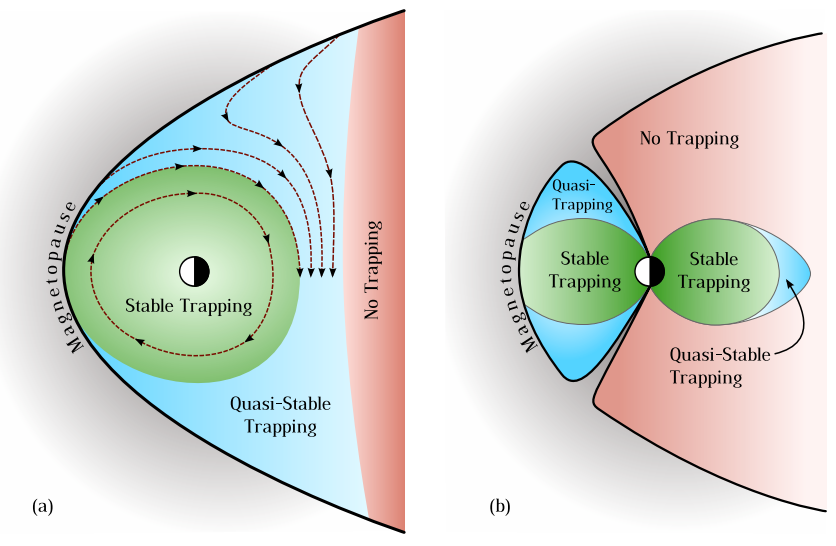

Fig. 1. Typical boundaries in the magnetosphere for trapped (green), quasi-trapped (blue), and untrapped (red) particles. (a) The equitorial plane with ion drift paths shown by dashed lines. (b) Noon-midnight plane (adapted from Roederer, 1970).

stably trapped, as the dayside extension is not great enough to allow the particles to contact the magnetopause. Outside of this region, the drift paths may extend enough to allow these pseudo-trapped or quasi-trapped particles to scatter on the magnetopause. (Roederer, 1967, 1970) These regions are represented by Fig. 1 .

The limit of stable trapping can exist in the region that will be discussed, near local midnight and with a radial distance between $6 R_{E}$ and $9 R_{E}$. Polar observes butterfly distributions both well inside and well outside this boundary. Outside of the surface that is created by the boundary of stable trapping, electrons and ions exist with a specific pitch angle in which the drift paths will no longer be closed around the earth, but extend into the magnetopause and will be subject to scattering processes that will scatter the particles away from their initial drift shell (Roederer, 1970). In this region of quasitrapping, charged particles with pitch angles near $90^{\circ}$ cannot orbit the earth in stable drift paths, but more field-aligned particles, which are not as affected by drift shell splitting, may remain in stable drift paths. It can be shown through particle modeling that the outer regions of Polar's observations of the nightside equatorial plane are subject to the differential drift paths created in the quasi-trapping region.

Plasma entry into the magnetopause has been simulated with magnetohydrodynamic (MHD) models (e.g., Walker et al., 2001). MHD simulations have also been used to study solar energetic particle entry during storms (Richard et al., 2009) and plasma sheet particles propagating to the highlatitude inner magnetosphere (Ashour-Abdalla et al., 2005). The modeling runs presented here are an effort to source nightside equatorially mirroring particles near geostationary distances observed by Polar. This is done by using a fully three-dimensional particle tracer in simulated magnetic and electric fields.

\section{Instrumentation}

Energetic ion detection onboard the Polar spacecraft is completed through the Imaging Proton Spectrometer (IPS). The instrument consists of nine sensors that rotate azimuthally with the spin axis of the satellite. In total, the instrument can detect ions with a $180^{\circ}$ polar $\times \sim 12^{\circ}$ azimuthal field of view. In a $96 \mathrm{~s}$ integrated observation, IPS detects a full sky image at sixteen different energy bands with central energies ranging from $15.6 \mathrm{keV}$ up to $1.51 \mathrm{MeV}$. The magnetic field direction, as measured onboard the Polar satellite (Russell et al., 1995), can then be used to determine the pitch angles of the protons detected by IPS. In a similar manner, the Imaging Electron Spectrometer (IES) can detect electrons with energies ranging from $20 \mathrm{keV}$ to $400 \mathrm{keV}$, with a $180^{\circ}$ polar $\times \sim 20^{\circ}$ azimuthal field of view.

\section{Presence of butterfly PADs in the nightside magnetosphere}

The butterfly PAD is a nearly permanent feature of certain regions of the magnetosphere. Polar was launched in 1996 with a $1.8 R_{E}$ perigee and a $9 R_{E}$ apogee over the northern pole, which then precessed southward, pushing the equatorial crossings further away from the earth. Over the next six years, Polar crossed the equatorial plane at radial distances between $3 R_{E}$ and $9 R_{E}$. From the years 1999 through 2002, the pitch angle distributions were studied for 384 equatorial crossings in the nightside of the magnetosphere. The local time ranged from $21 \mathrm{~h}$ through midnight and on to $3 \mathrm{~h}$. The survey was conducted for the months of August, September, and October for each year, as these were the months that the orbital apogee was near local midnight. The presence of the butterfly PAD increased in frequency outside of $5 R_{E}$. For example, in between $5.5 R_{E}$ and $9.5 R_{E}$, a butterfly PAD was observed in more than $85 \%$ of the equatorial crossings. The frequency of occurrence of the butterfly PAD is shown in Table 1 for the equatorial range of Polar in $0.5 R_{E}$ bins.

\section{A closer look at PADs from two equatorial crossings}

Pitch angle distributions are shown as functions of time for two specific days: 26 October 1999 and 12 September 2000. These days were chosen to demonstrate certain characteristics that are commonly observed by the IES and IPS on Polar. The features in the data that will be discussed on these two days are generally not unique, but were chosen for their clarity.

For the following two events, the apogee of the orbit was near local midnight. For both events, the satellite passed through the magnetic equatorial plane at a radial distance between $6 R_{E}$ and $7 R_{E}$, as it was moving along an outbound path, from the Southern to the Northern Hemisphere. Figure 2 demonstrates a typical path through the noon-midnight 
Table 1. The frequency of Butterfly PADs is characterized by separating all of the crossing statistics into $0.5 R_{E}$ bins and calculating the occurrence statistics as a function of radial distance.

\begin{tabular}{lcccccccccccc}
\hline & \multicolumn{1}{c}{ Number of occurrences within each $0.5 R_{E}$ bin with center at radial distance $\left(R_{E}\right)$} \\
Radial distance $\left(R_{E}\right)$ & 4.25 & 4.75 & 5.25 & 5.75 & 6.25 & 6.75 & 7.25 & 7.75 & 8.25 & 8.75 & 9.25 & 9.75 \\
\hline Butterfly & 0 & 9 & 4 & 40 & 38 & 19 & 17 & 31 & 23 & 19 & 59 & 31 \\
No butterfly & 7 & 22 & 10 & 6 & 6 & 1 & 3 & 4 & 1 & 2 & 9 & 11 \\
\hline Total crossings & 7 & 31 & 14 & 46 & 44 & 20 & 20 & 35 & 24 & 21 & 68 & 42 \\
Butterfly occurence $(\%)$ & 0.0 & 29.0 & 28.6 & 87.0 & 86.4 & 95.0 & 85.0 & 88.6 & 95.8 & 90.5 & 86.8 & 73.8 \\
\hline
\end{tabular}

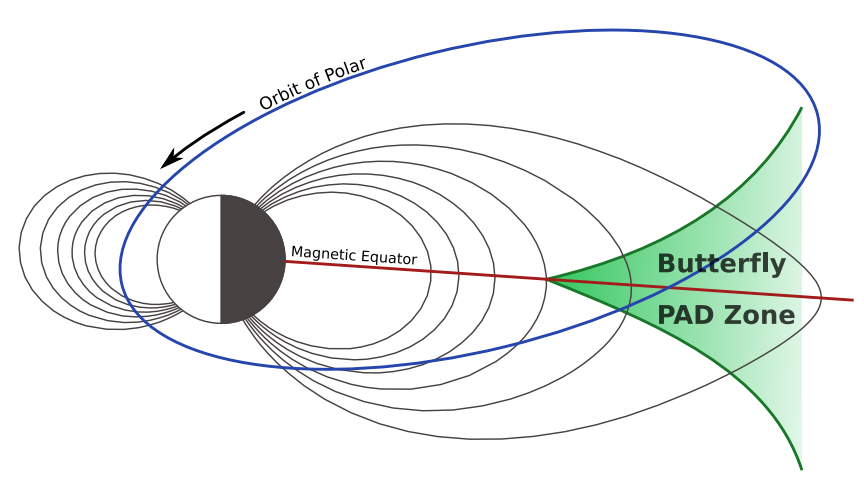

Fig. 2. Typical orbit for Polar in the fall of 1999. The satellite moves outward through the zone of typical butterfly PADs.

plane. Before Polar reached a region of butterfly PAD, it passed through the energetic radiation belts where PADs are peaked at $90^{\circ}$. As it left the butterfly region, the satellite was usually far enough north of the equatorial plane that the measured fluxes were observed to fall off significantly.

\subsection{October 1999}

Electron butterfly PADs were observed by the IES across all available energy channels as Polar crossed the nightside magnetic equatorial equator as displayed in Fig. 3. Additionally, the timing of the onset of the butterfly PADs, as well as the ratio of the peak flux in the PAD to that at the minimum at $90^{\circ}$ are observed to remain approximately constant throughout the energy range of the IES.

Figure 4 displays the pitch angle distributions of energetic ions as observed by Polar as a function of time from 11:30 UT until 14:30 UT. In all, sixteen energy channels are displayed with the title of each indicating the central energy of the channel. There is contamination from the sun that is observed in the lowest energy channel occurring with pitch angles under $20^{\circ}$.

For ions with energies between $70 \mathrm{keV}$ and $439 \mathrm{keV}$, the pitch angle plots from IPS indicate a pronounced butterfly PAD when Polar was near the nightside magnetic equatorial plane from 12:30 UT until 13:30 UT. The specific onset and duration of the butterfly PAD is dependent upon the observed energy channel. In general, the butterfly shape is observed in the PAD first in the more energetic channels. In orbital space, this earlier onset corresponds to a slightly lower L-shell, as the spacecraft was outbound during this period. As the energies of the ion channels increase, the ratio of the maximum flux (usually at pitch angles near $40^{\circ}$ and $140^{\circ}$ ) to the locally mirroring minimum becomes less pronounced. For the highest three energy channels, the incident fluxes are low enough such that butterfly PADs cannot be accurately determined.

Pancake PADs are also observed in addition to the butterfly PADs at the same time, but the pancake PADs occur only at lower energy levels. The lowest four energy channels have a PAD that is peaked at $90^{\circ}$, while the $51 \mathrm{keV}$ channel simultaneously shows both butterfly and pancake PAD components from 13:20 UT until 13:30 UT. This can indicate two distinct populations that co-exist in the the nightside magnetic equatorial region. Here, less energetic ions are dominated by a pancake PAD with a maximum flux for $90^{\circ}$ pitch angle ions. In the more energetic channels of the IPS instrument, the opposite occurs where more field aligned ions have the dominant fluxes.

\subsection{September 2000}

Like the electrons observed in the 26 October event, Fig. 5 shows that IES detects butterfly PADs across the instrument's entire energy range, with the $90^{\circ}$ dropout occurring at almost identical times and relative intensities. This is a behavior that is not observed with the IPS detection of the protons.

Figure 6 displays the pitch angles of energetic ions as observed by Polar as a function of time from 05:00 UT until 07:00 UT. In all, sixteen energy channels are displayed with the title of each indicating the central energy of the channel. Like the 26 October event, IPS measures simultaneous high energy butterfly and low energy pancake PADs.

Also similarly, there is a $90^{\circ}$ pitch angle peak that is nested within a butterfly PAD in the $38 \mathrm{keV}$ channel between approximately 06:00 UT and 06:30 UT. The plots also show that when these sources with peaks at pitch angles of $90^{\circ}$ appear in the lower energy channels, they have a V-shape in the plots. In a temporal sense, they are first observed by the 
Polar IES Pitch Angle Plots (26 October 1999)
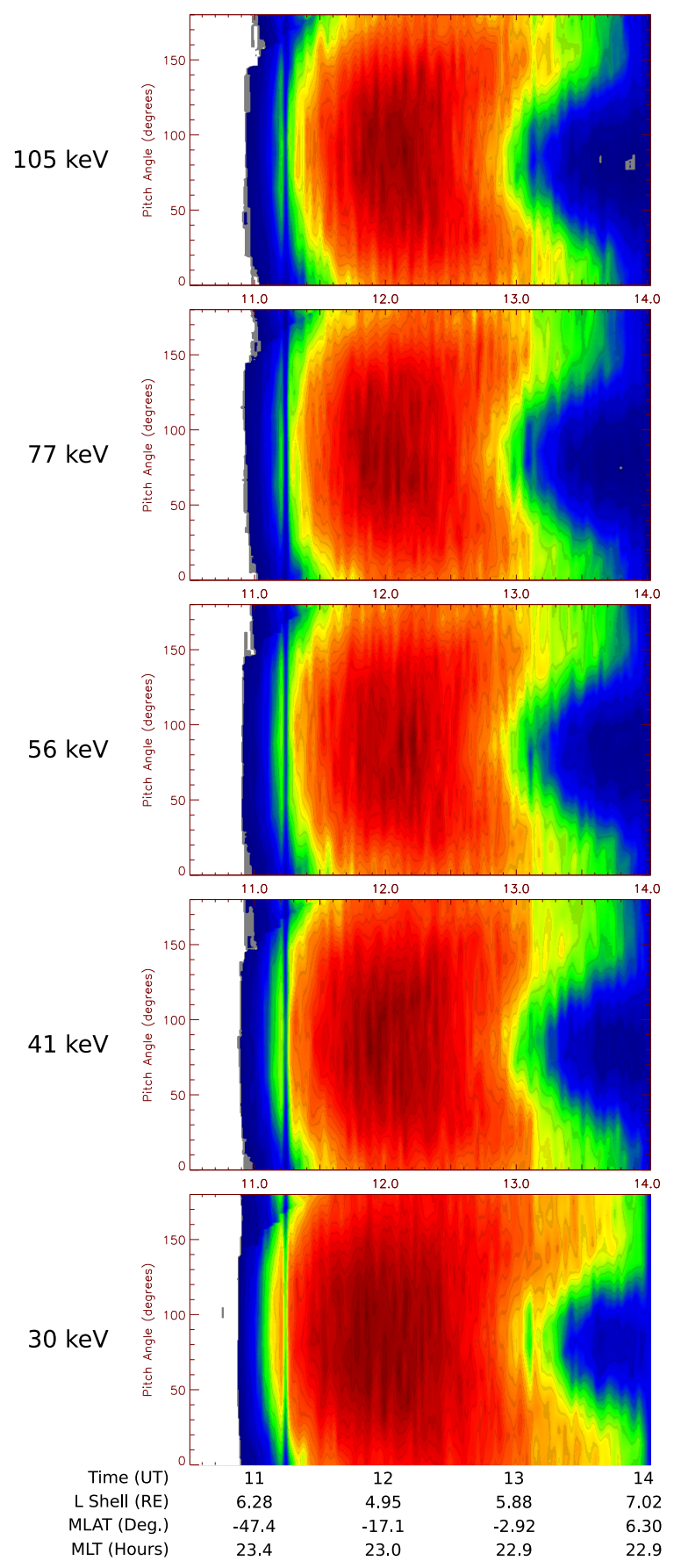
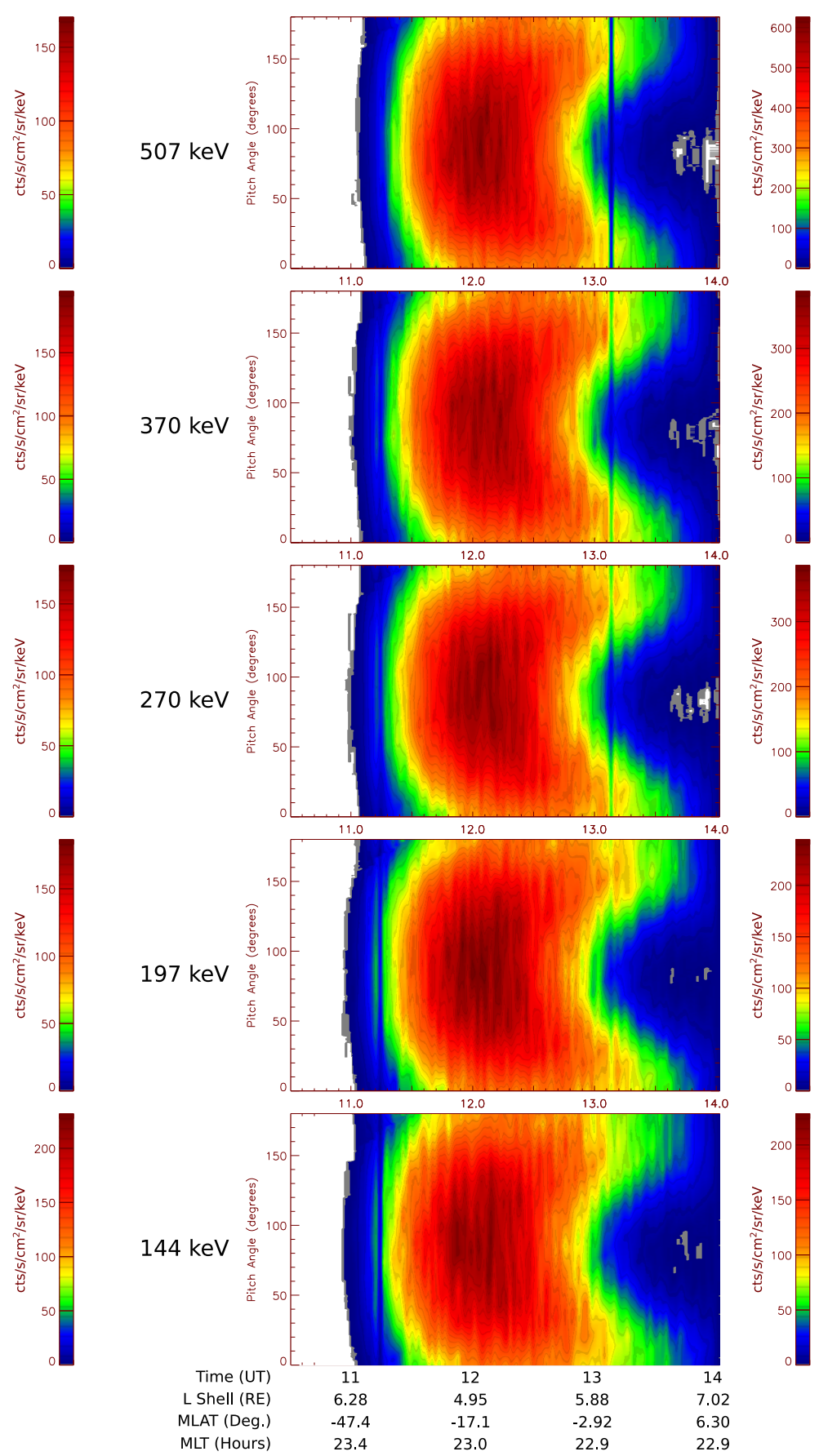

Fig. 3. Electron pitch angle intensities plotted as a function of time for ten different energy levels from $20 \mathrm{keV}$ to $400 \mathrm{keV}$. Observations are from 10:30 UT until 14:00 UT on 26 October 1999. The energies given to the left of each plot represent the nominal energy thresholds for each channel.

satellite at $90^{\circ}$ and expand to more field aligned pitch angles as time progresses. In a spatial sense, the satellite observes the $90^{\circ}$ pitch angle particles closer to the earth than particles of other pitch angles, as it is on an outbound orbit as shown in Fig. 2. 
Polar IPS Pitch Angle Plots (26 October 1999)
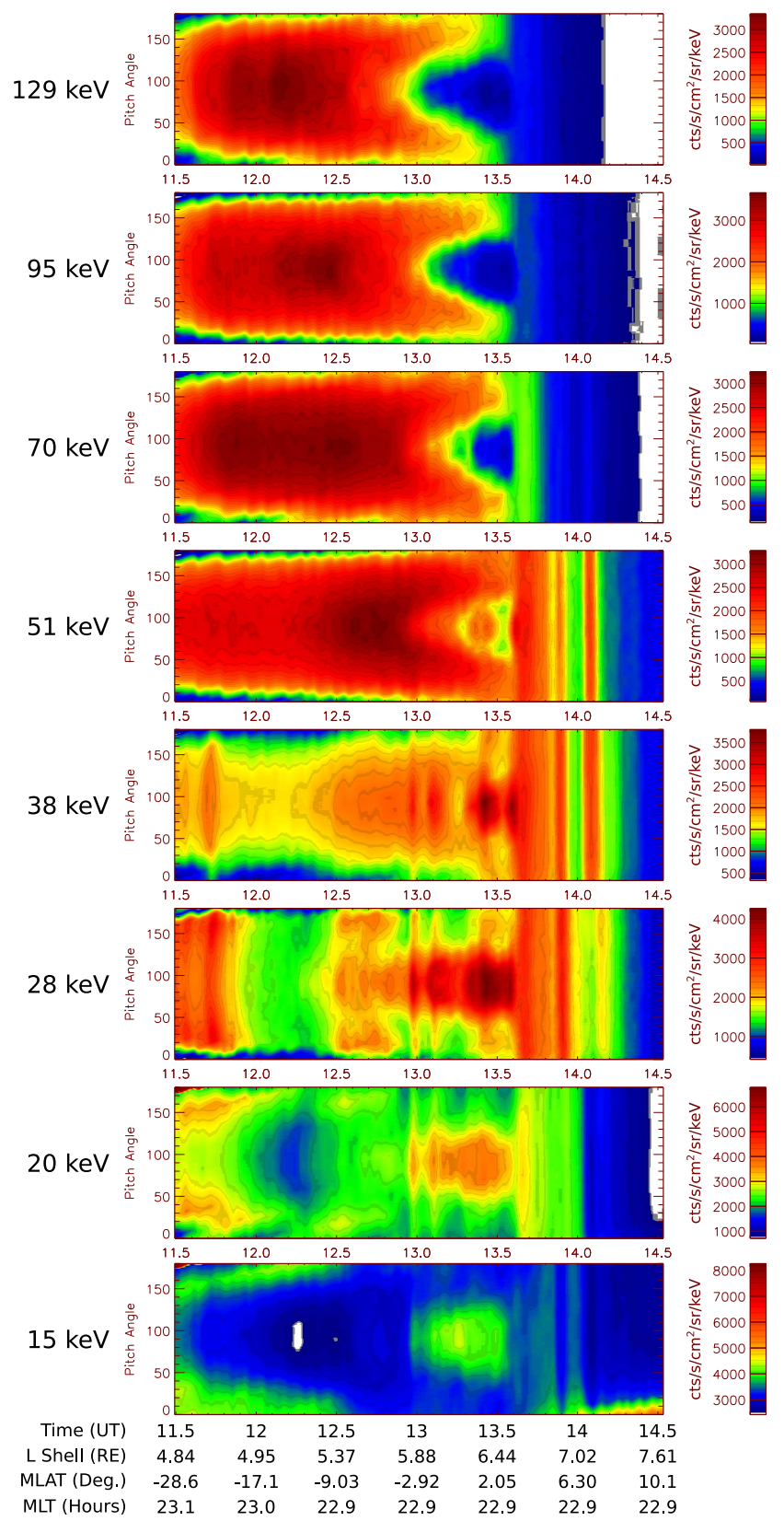
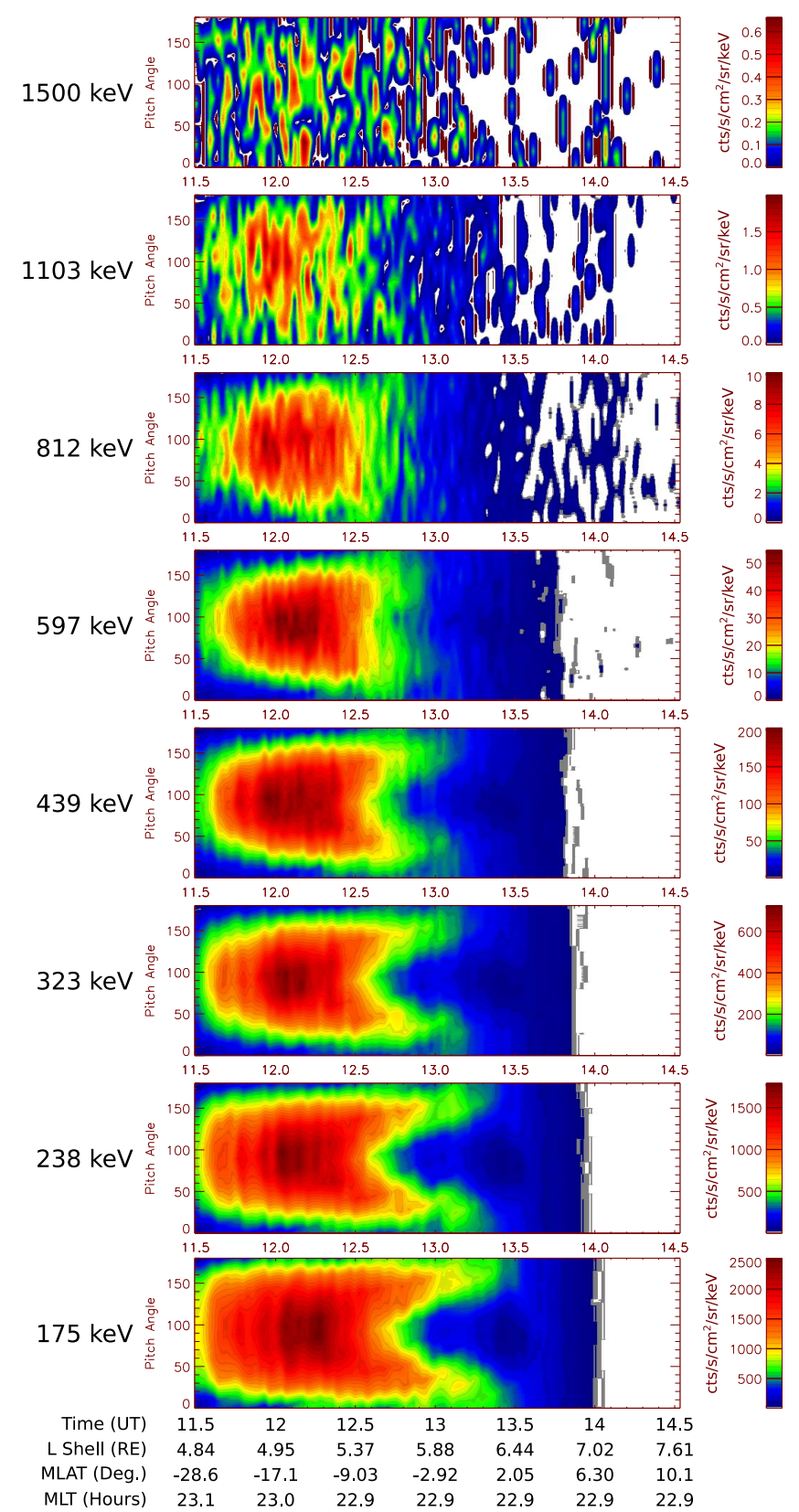

Fig. 4. Ion pitch angle intensities plotted as a function of time for sixteen different energy levels. Observations are from 11:30 UT until 14:30 UT on 26 October 1999. The final $30 \mathrm{~min}$ of the lowest energy channel shows contamination from the sun for pitch angles less than $20^{\circ}$ and is not part of the local proton PAD. The energies given to the left of each plot represent the nominal energy thresholds for each channel.

\section{Modeling charged particles in magnetosphere}

A particle's motion in the magnetosphere can be concisely described by the Lorentz force,

$\mathbf{F}=m \frac{d \mathbf{v}}{d t}=q(\mathbf{E}+\mathbf{v} \times \mathbf{B})$ for a particle with mass $m$, charge $q$, and velocity $\mathbf{v}$ existing in an electric field $\mathbf{E}$ and magnetic field $\mathbf{B}$. In a simple dipole magnetic field, electrons or ions will exhibit well-behaved motions of gyration, bounce, and drift. When a charged particle is modeled in a realistic magnetosphere, this relatively simple equation can produce complex orbital paths. If the 

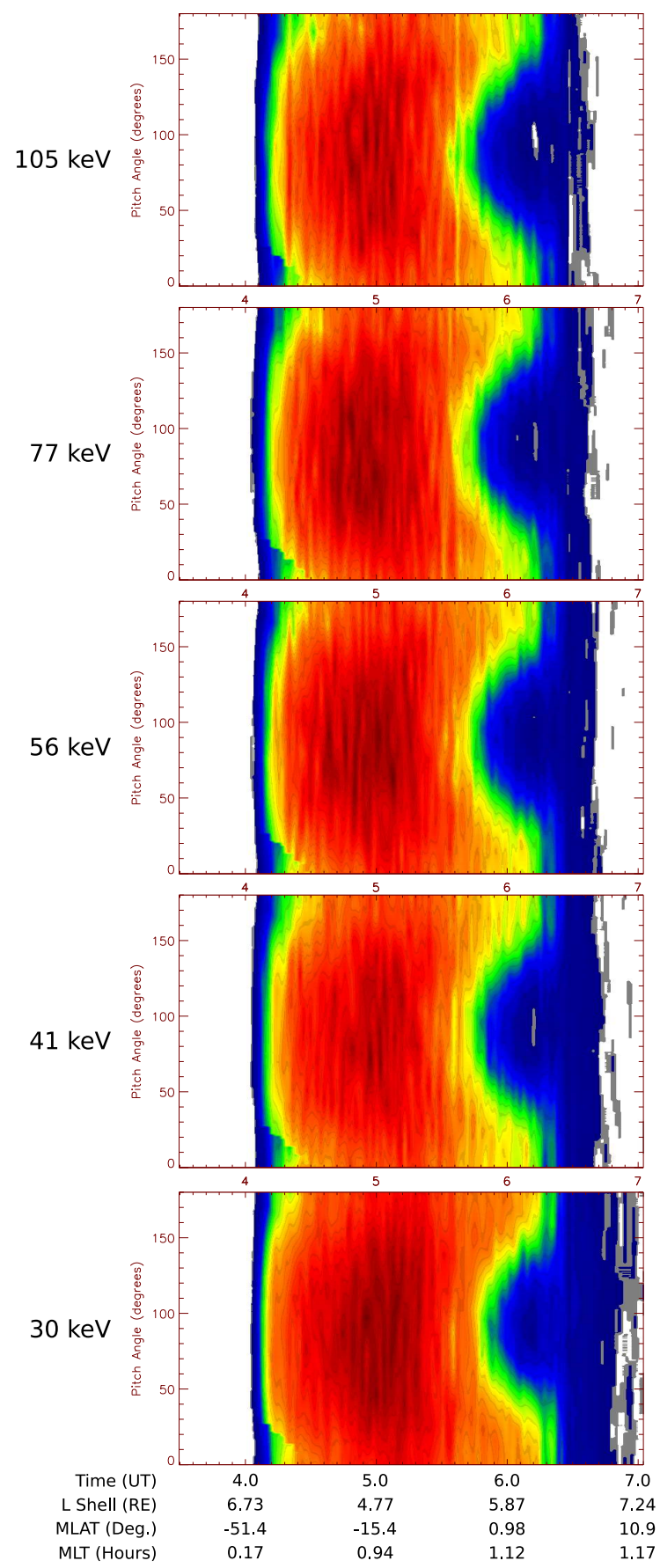
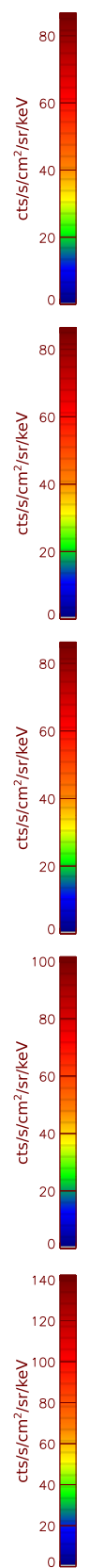

$507 \mathrm{keV}$
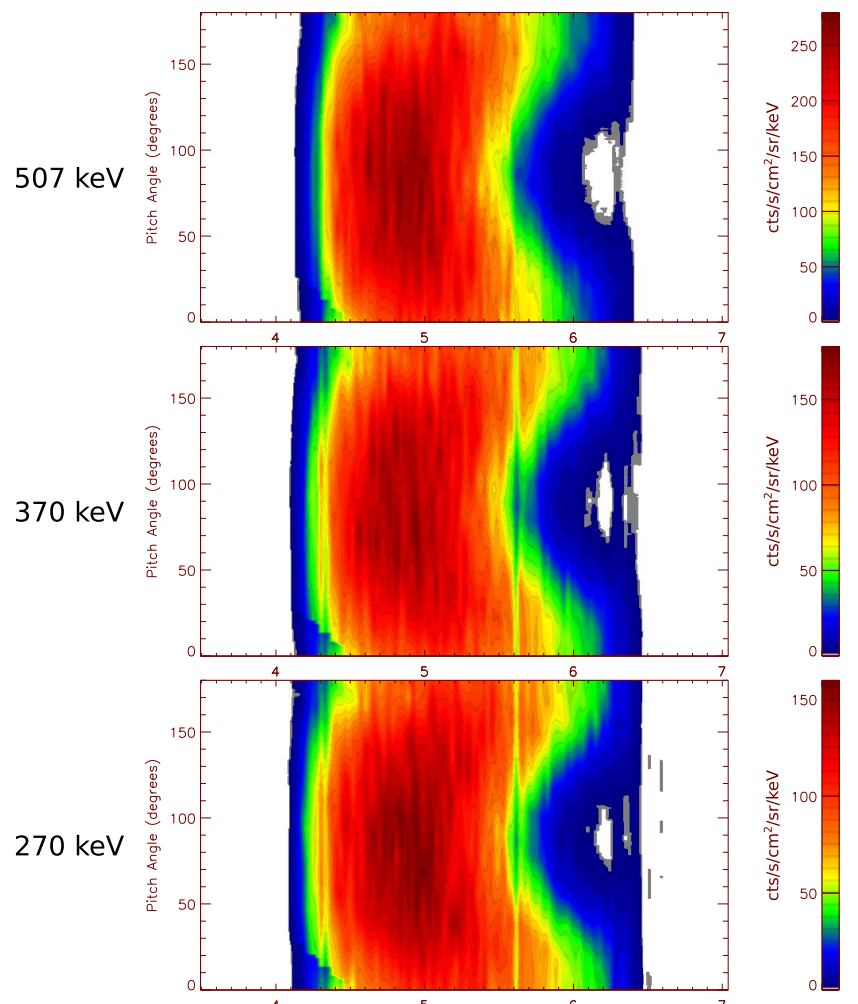

$197 \mathrm{keV}$
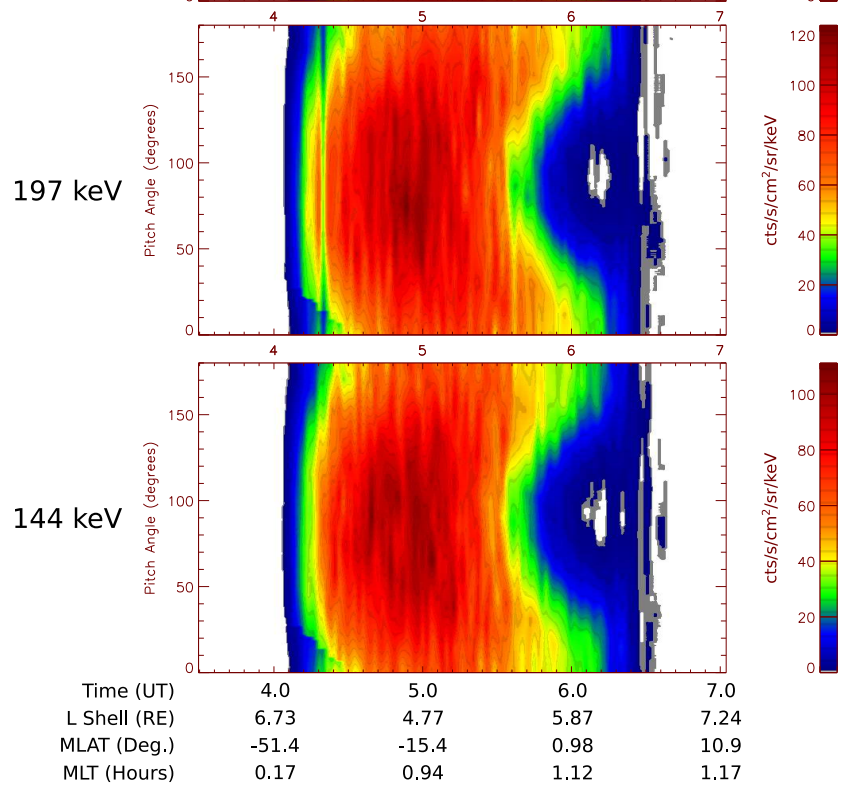

Fig. 5. Electron pitch angle intensities plotted as a function of time for ten different energy levels from $20 \mathrm{keV}$ to $400 \mathrm{keV}$. Observations are from 03:30 UT until 07:00 UT on 12 September 2000. The energies given to the left of each plot represent the nominal energy thresholds for each channel.

conservation of the adiabatic invariants is assumed, then the motion can still be accurately predicted through a combination of simple forces. It frequently occurs however, that a charged particle outside of geostationary orbit will drift in a region of stretched and distorted magnetic field geometry. At this point, even the first adiabatic invariant cannot be 
Polar IPS (12 September 2000)
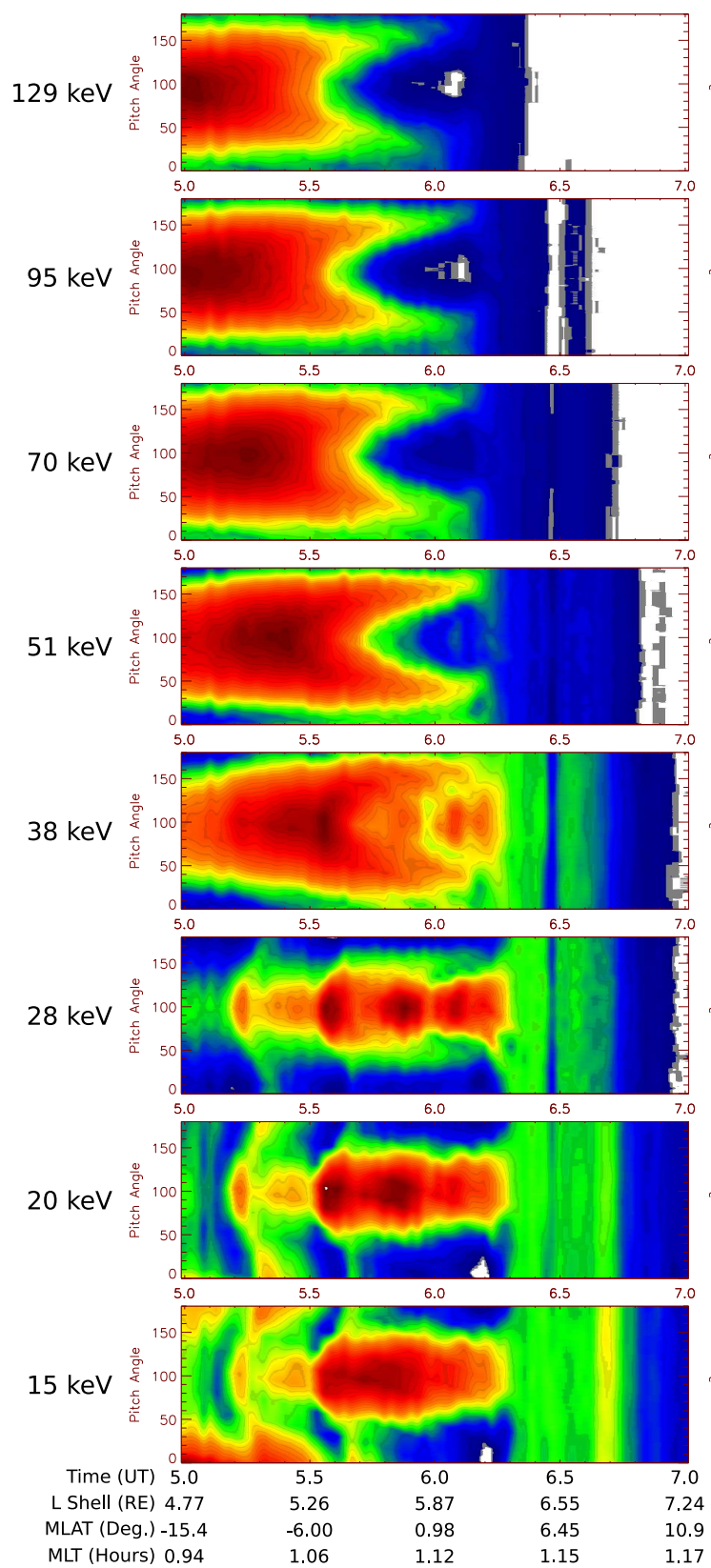
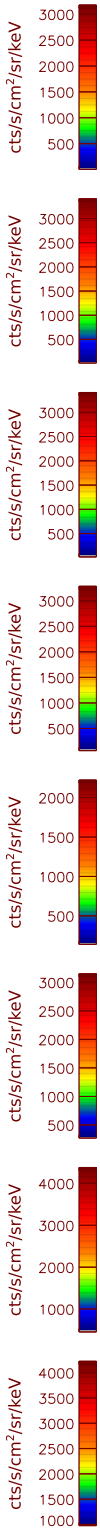
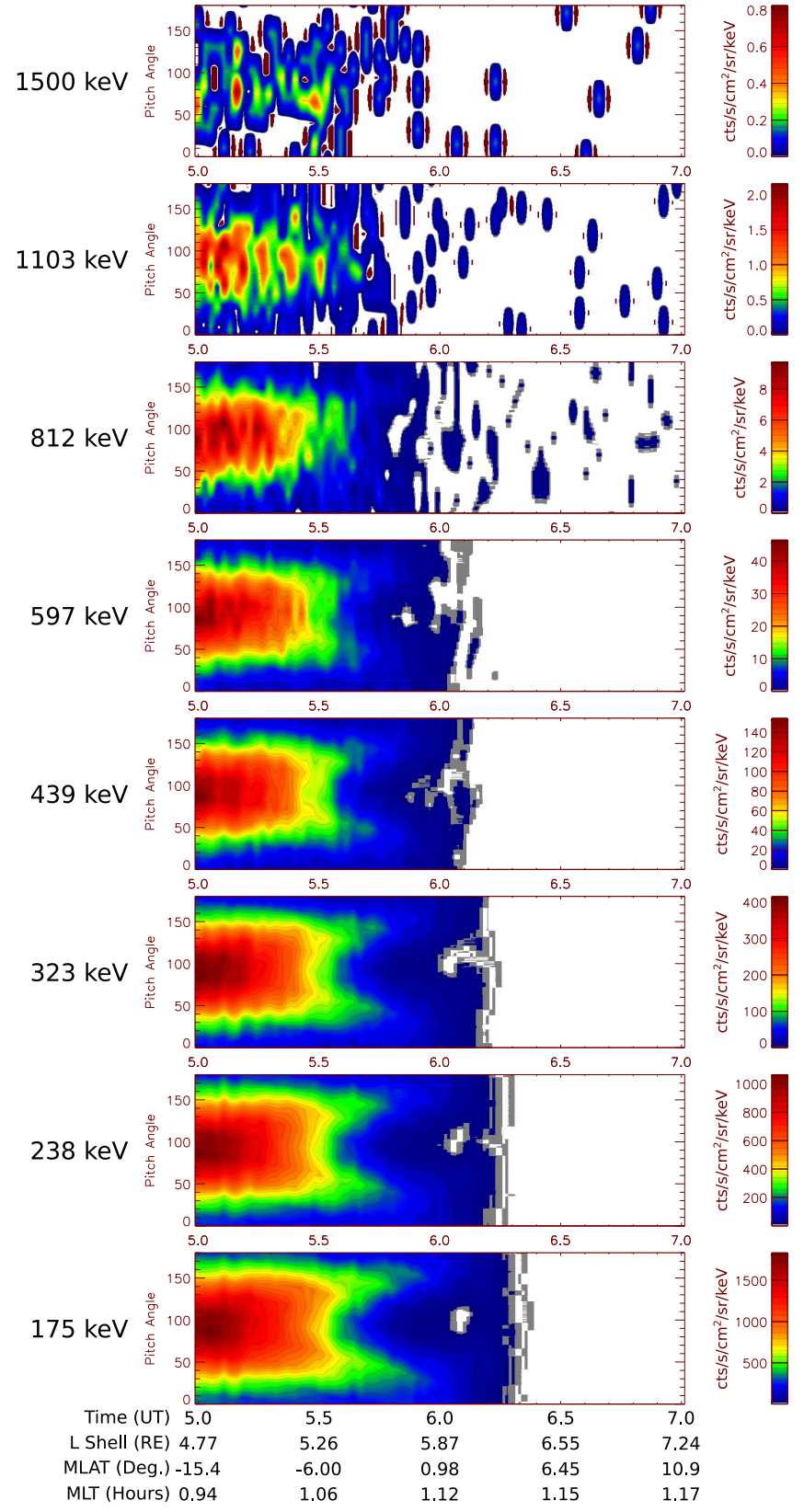

Fig. 6. Ion pitch angle intensities plotted as a function of time for sixteen different energy levels. Observations are from 05:00 UT until 07:00 UT on 12 September 2000. The energies given to the left of each plot represent the nominal energy thresholds for each channel.

assumed to be conserved and a modeler is left with only the Lorentz force, a differential equation which must be solved numerically.

For all of the simulations presented herein, the differential equation from the Lorentz force was fully relativistic and numerically solved using a stepsize of one percent of the gyro orbit. For each step, the velocity of the particle was numerically calculated. Using the velocity vector and the time step, the particle was then translated to a new location. The process was then repeated until the simulation was finished.

A code for tracing particles through a magnetosphere was created for IDL that allows for 3-dimensional particle tracing without the use of the guiding center approximation. This is important as the stretched magnetic field topology is not always ideal for the use of a guiding center approximation. Given a particle's initial mass, charge, energy, location, pitch 
26 Oct. 1999 OMNI 1 AU IMF and Solar Wind Plasma Data (1 Minute Resolution)

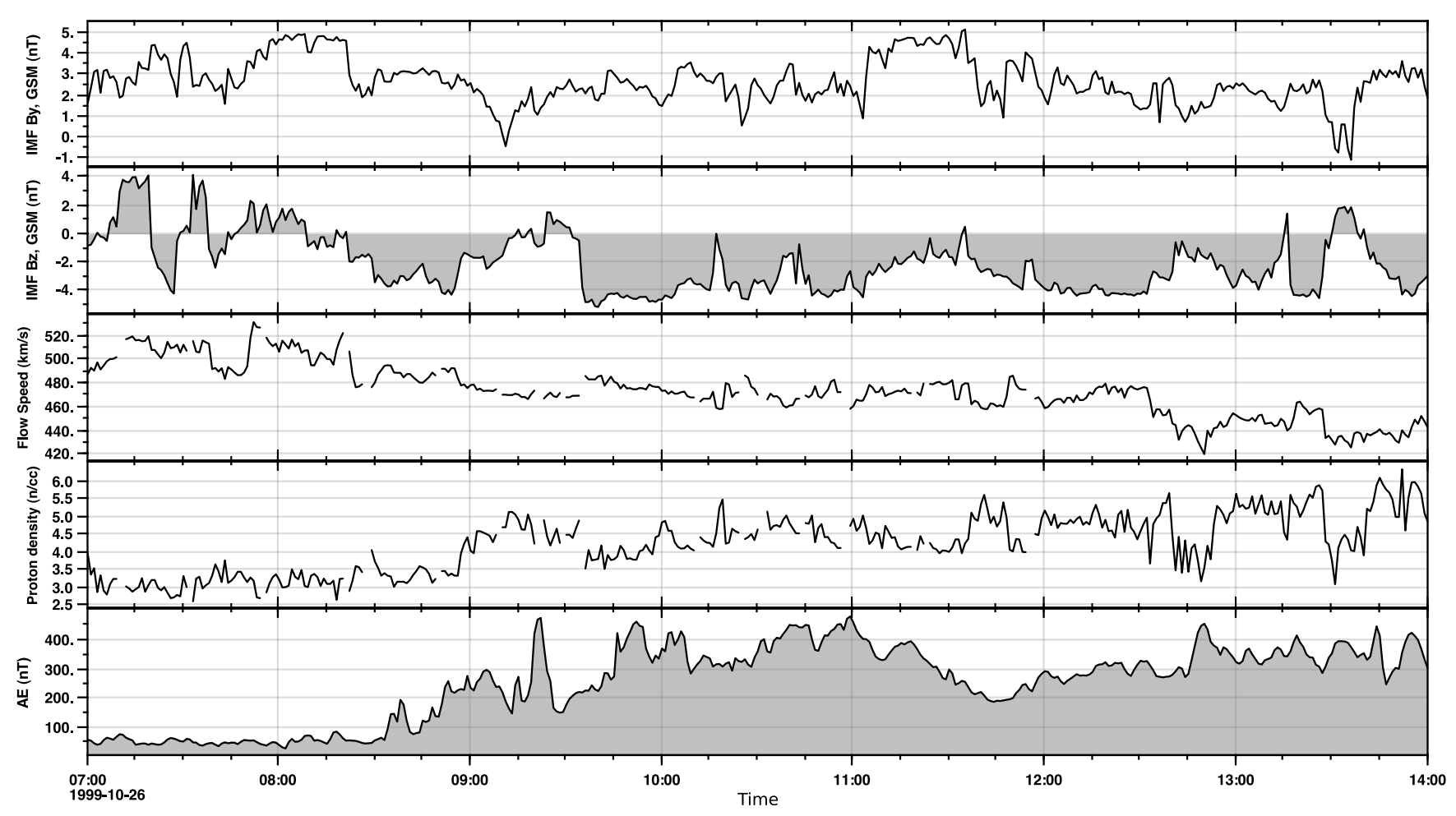

Fig. 7. OMNI solar wind data propagated to $1 \mathrm{AU}$ from 07:00 to 14:00 on 26 October 1999. From the top panel, measurements include IMF $B_{y}$, IMF $B_{z}$, plasma flow speed, ion density, and the AE index.

angle, and gyrophase, the tracer can simulate the temporal progression of the particle in the magnetosphere. By default the step size of the trace is one percent of the gyro-orbit and a sixth order Runge-Kutta method is used to numerically solve the differential equation. However, both of these can be modified to expedite the computation process.

Using the Tsyganenko TS05 magnetic field model (Tsyganenko, 1995; Tsyganenko and Sitnov, 2005) and the VollandStern electric field model (Stern, 1975; Volland, 1978) to simulate the magnetosphere, a fairly realistic model can be created to trace the drift, bounce, and gyration of charged particles at varying energies, charges, pitch angles, and masses.

\subsection{The Volland-Stern electric field model}

The Voland-Stern Electric field Model is a combination of the corotation electric potential and the dawn-to-dusk convection potential. The intensity of the electric potential $\phi$, is empirically fitted to scale with the $K_{p}$ index. For any point in space, the electric field $\mathbf{E}$ can be found though calculating the gradient of the surrounding electric potential such that, $\mathbf{E}=-\nabla \phi$. Although the model does not incorporate a magnetopause, one may be inferred when the model is used in conjunction with the TS05 model. And it is this electric field that is put into the Lorentz force to yield an overall force vector. The model only yields equatorial electric potentials. For locations off of the equator, an approximation must be used. The magnetic field line is traced to the equator and it is this equatorial electric potential that is transposed to the new point keeping the parallel components of the electric field at zero.

\subsection{The Tsyganenko-Sitnov 2005 magnetic field model}

This magnetic field model is semi-empirically based by fitting parameters to numerous satellite data sets. The input parameters are solar wind (SW) dynamic pressure, the Dst index, as well as the $y$ - and z-components of the interplanetary magnetic field (IMF). The model will also determine the location of the earth's dipole axis given the time and date. This model will return a full three-dimensional magnetic field vector at any point in the earth's magnetosphere.

\section{Single particle motion modeled in a simulated magnetosphere}

Using the aforementioned models, we can trace particles to determine where they will go, which may be used to 


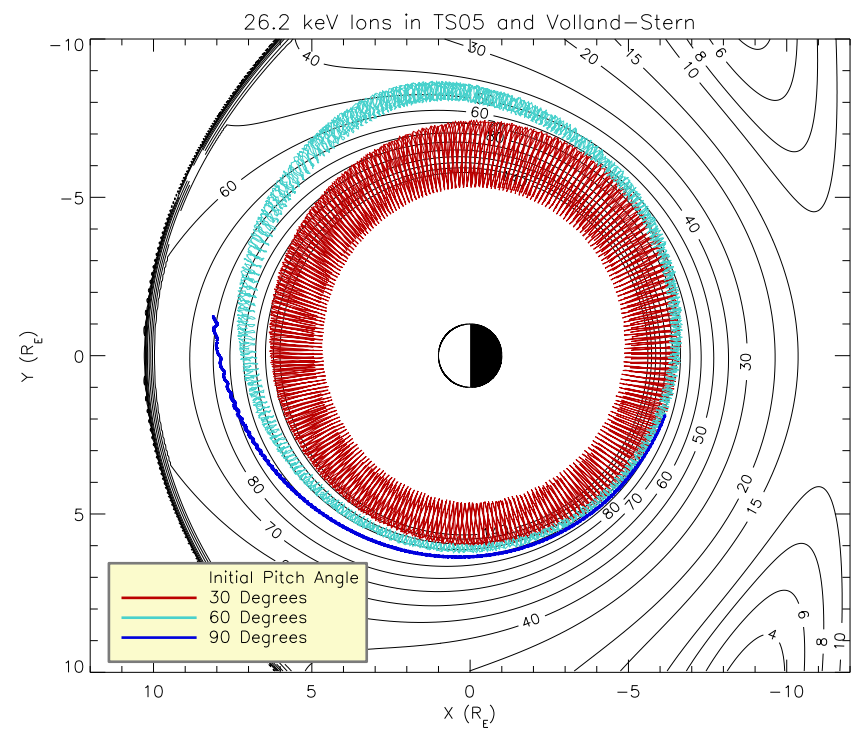

Fig. 8. $26.2 \mathrm{keV}$ ions simulated forward in time beginning at Polar's position at 13:27 UT on 26 October 1999 for initial pitch angles of $30^{\circ}$ and $60^{\circ}$, which are stably trapped, and $90^{\circ}$, which is unstable.

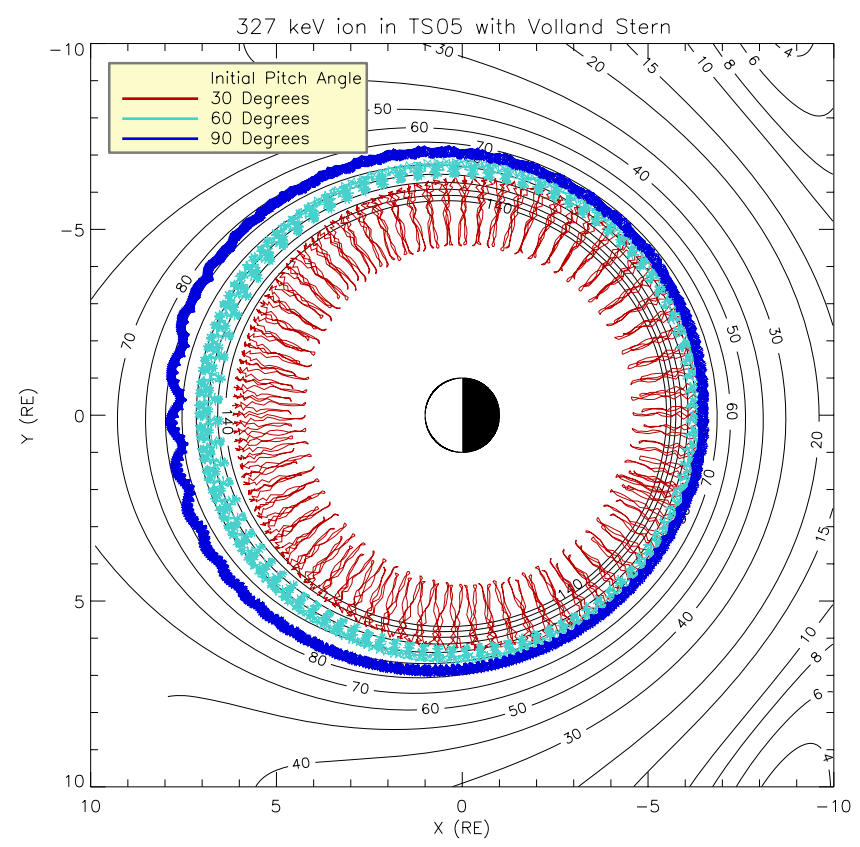

Fig. 9. $327 \mathrm{keV}$ ions simulated forward in time beginning at Polar's position at 13:27 UT on 26 October 1999 for initial pitch angles of $30^{\circ}, 60^{\circ}$, and $90^{\circ}$. All three of the modeled pitch angles are in stably trapped drift orbits.

determine stability, or from where they came, used to determine a particle's origins. Energetic ions were simulated to progress along their bounce and drift paths forward in time to determine if the particles observed by the IPS sensor can drift

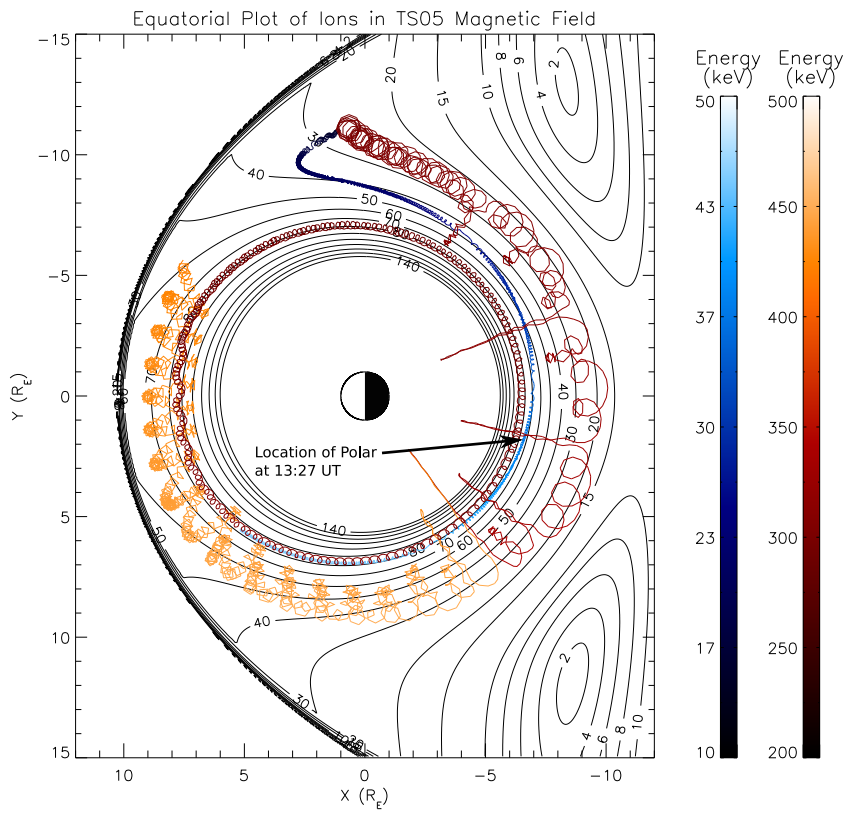

Fig. 10. $15 \mathrm{keV}$ and $300 \mathrm{keV}$ ions simulated forward in time beginning at $x=1.2 R_{E}, y=-11.0 R_{E}$, and $z=1.3 R_{E}$ with magnetospheric conditions at 13:27 UT on 26 October 1999 with initial pitch angle of $90^{\circ}$. Additionally, a $300 \mathrm{keV}$ ion started at Polar's location and is shown to be stably trapped. The left (blue) color bar is associated with the low energy ion, and the right (red) color bar is associated with the high energy ions.

around the earth on closed paths. Ions were simulated at $30^{\circ}$, $60^{\circ}$, and $90^{\circ}$ initial pitch angles with energies of $26.2 \mathrm{keV}$ and $327 \mathrm{keV}$ for the 26 October 1999 observations. These energies correspond to the energies of channels toward the low and high ends of the IPS instrument. The particles were traced so that a full drift path could be completed, if possible. All of the following tracing were made in a magnetosphere modeled at 13:27 UT, which is the approximate time of the strongest low energy intensification observed by IPS that day. The input parameters for the models at this time are as follows: $n_{s w}=4.85 \mathrm{~cm}^{-3}, v_{s w}=473 \mathrm{kms}^{-1}, D_{s t}=-33$, $B_{y}(\mathrm{IMF})=2.81 \mathrm{nT}, B_{z}(\mathrm{IMF})=-3.73 \mathrm{nT}$, and $K_{p}=3$. A one minute resolution time time history of the IMF and solar wind plasma data along with the AE index from 07:00 until 14:00 UT can be seen in Fig. 7 .

Figures 8 and 9 represent traces of ions that originate at Polar's location at 13:27 UT, which in GSM coordinates is: $x=-6.17 R_{E}, \mathrm{y}=1.85 R_{E}$, and $z=-0.34 R_{E}$. The $327 \mathrm{keV}$ figure suggests that the drift paths are closed and stable for all pitch angles, while the $26.2 \mathrm{keV}$ plot indicates that the drift orbits are stable for $30^{\circ}$ and $60^{\circ}$ pitch angles, but the $90^{\circ}$ particle is not stably trapped.

If the $26.2 \mathrm{keV}$ ions with a $90^{\circ}$ pitch angle are not stably trapped, it is important to determine where these intensifications observed by Polar originate. A starting location 

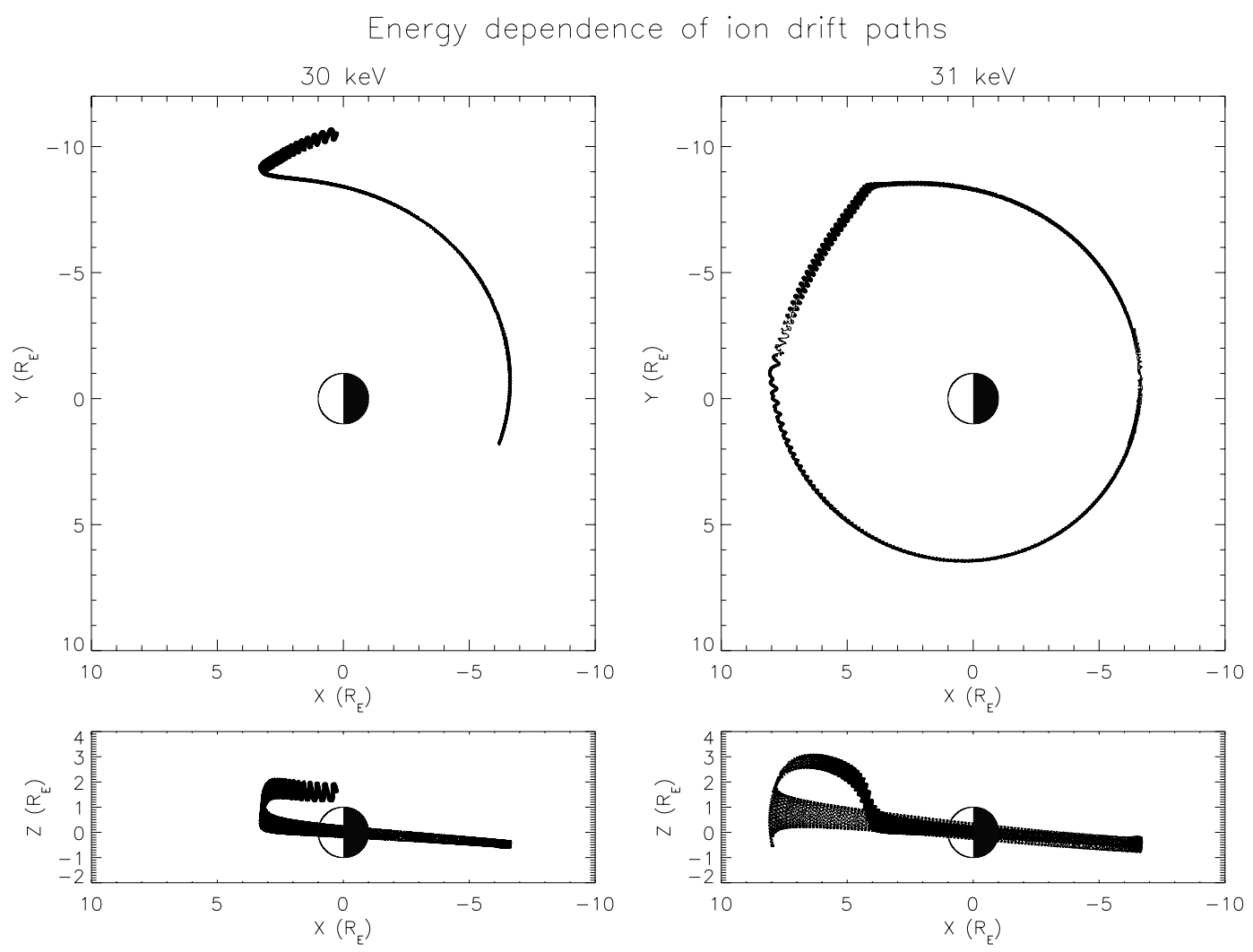

Fig. 11. Ions traced backward in time in a TS05 magnetic field and Volland-Stern electric field. A $30 \mathrm{keV}$ ion in the left column traces back to the dawn flank, while a $31 \mathrm{keV}$ ion in the right column is in a Shabansky orbit.

was first determined to be near the dawn flank of the magnetopause $\left(x=1.2 R_{E}, y=-11.0 R_{E}\right.$, and $\left.z=1.3 R_{E}\right)$, and then a $15 \mathrm{keV}$ ion was modeled forward in time and the path is shown in Fig. 10 with the orbit of Polar during the low energy intensification from 13:00 UT until 13:36 UT on 26 October 1999. This path is indicated by the blue line. As the ion drifts to a location near the position of Polar, it moves radially inward an is energized to an energy of $30 \mathrm{keV}$. So the intensification of lower energy $90^{\circ}$ pitch angle particles that is measured by Polar in the nightside equatorial region can originate from the dawn side flank, allowing the magnetopause to be the source of these particles.

As shown before, the $300 \mathrm{keV}$ ion in the vicinity of Polar is on a stably trapped orbit. If there is a flank source, the fact that Polar only observes a low energy intensifications cannot be an indication of the energy spectrum of the source itself. Figure 10 also shows a $300 \mathrm{keV}$ ion that started at the same dawn source location as the $15 \mathrm{keV}$ ion. It drifts across the nightside, but at a larger radial distance. As the energizing effect of the electric field is diminished for this higher energy particle, it cannot penetrate as far inward as the $15 \mathrm{keV}$ ion. It is believed that the inconsistent bounce motion of this $300 \mathrm{keV}$ ion is not a modeling defect, but a consequence of the first invariant violation which is due to large gyro radii in a highly stretched nightside magnetic field.

\section{A source population}

The particle trajectories in Fig. 10 show that the equatorially mirroring ions observed by Polar in the less energetic channels can be traced back to the dawn flank of the magnetosphere. The particular entry point of these ions into the flank is subject to fine variations in both the particle's energy and electric field strength. Figure 11 shows two ions traced backward in time with a starting location that is at Polar's position in the nightside at 13:27 UT, using the same 26 October 1999 input conditions. The particles have initial nightside energies of 30 and $31 \mathrm{keV}$. Despite the nominal energy difference of only $1 \mathrm{keV}$, the modeled origins of each particle are noticeably different. The more energetic particle display's a trapped drift shell that moves into an off-equatorial bounce into the Northern Hemisphere on the dayside, while the less energetic particle originates from the dawn flank. The differences in these drift paths arise from the fact that the $\boldsymbol{E} \times \boldsymbol{B}$ drift is independent of energy, and the gradient- $B$ drift is not. The more energetic $31 \mathrm{keV}$ ion has a gradient- $B$ drift force that is strong enough to keep its drift shell closed around the dawn terminator, while the $30 \mathrm{keV}$ ion cannot be on a closed drift shell.

Such sensitivity to small deviations in the electric field models or particle energies limits the ability to pinpoint exact 
locations along the magnetopause from where these particles are originating. As long as small errors exist in the magnetic and electric field models, it may be difficult or impossible to do this using a Lorentz force particle tracing technique. However, the important idea to keep in mind is that these particles are coming from the dawnside flank near the magnetopause. Because of this conclusion, a population of ions that is introduced along the dawnside can couple into the magnetosphere and be transported to the nightside.

The ion pitch angles presented in Figs. 4 and 6 both show ion intensifications that occur in the lower energy ranges. When the IPS detects an intensification, the ions with a pitch angle of $90^{\circ}$ are measured first, with more field aligned particles detected afterward. This process can be seen in the $28 \mathrm{keV}$ channel on the 26 October event beginning approximately at $12: 18 \mathrm{UT}$ and on the $20 \mathrm{keV}$ channel on the 12 September event beginning approximately at 05:12 UT.

If an isotropic population is introduced into the magnetosphere along the dawnside magnetopause, it would drift westward to the nightside. As a consequence of drift shell splitting, the original $90^{\circ}$ ions would drift to the nightside with the closest distance to the earth. The more field-aligned that the particle was in the initial location the more distant it will be when drifting around the nightside.

In both of these observations, the Polar spacecraft is moving along an outbound trajectory in the nightside equatorial region, as shown in Fig. 2. The earlier observations of the $90^{\circ}$ pitch angle ions corresponds to a lower L-shell value than when the more field aligned particles are observed. This pattern is indicative of an isotropic source on the dayside.

\section{Ion energy spectra}

During the butterfly PAD observed by Polar on 12 September 2000 , there is an additional population with a peak intensity at a $90^{\circ}$ pitch angle. Using the sixteen available energy channels on the IPS, it is possible to create energy spectra from the ion measurements. Figure 12 shows the observed ion spectra for populations with $30^{\circ}$ and $90^{\circ}$ pitch angles. The particles intensities were averaged over a half hour time interval from 05:48 UT until 06:18 UT.

The ions with $30^{\circ}$ and $90^{\circ}$ pitch angles are shown to be distinct populations with their own spectra, with the $90^{\circ}$ pitch angle particles dominating at low energies, but falling off more quickly than the $30^{\circ}$ pitch angle ions. It may then be concluded that the appearance of locally mirroring particles that are observed by Polar under $60 \mathrm{keV}$ are a population distinct from the butterfly distribution commonly observed in the nightside equatorial region.

\section{Conclusions}

Butterfly PADs are exemplified in the electrons at all energies measured by the IES with high frequencies of occurrence in

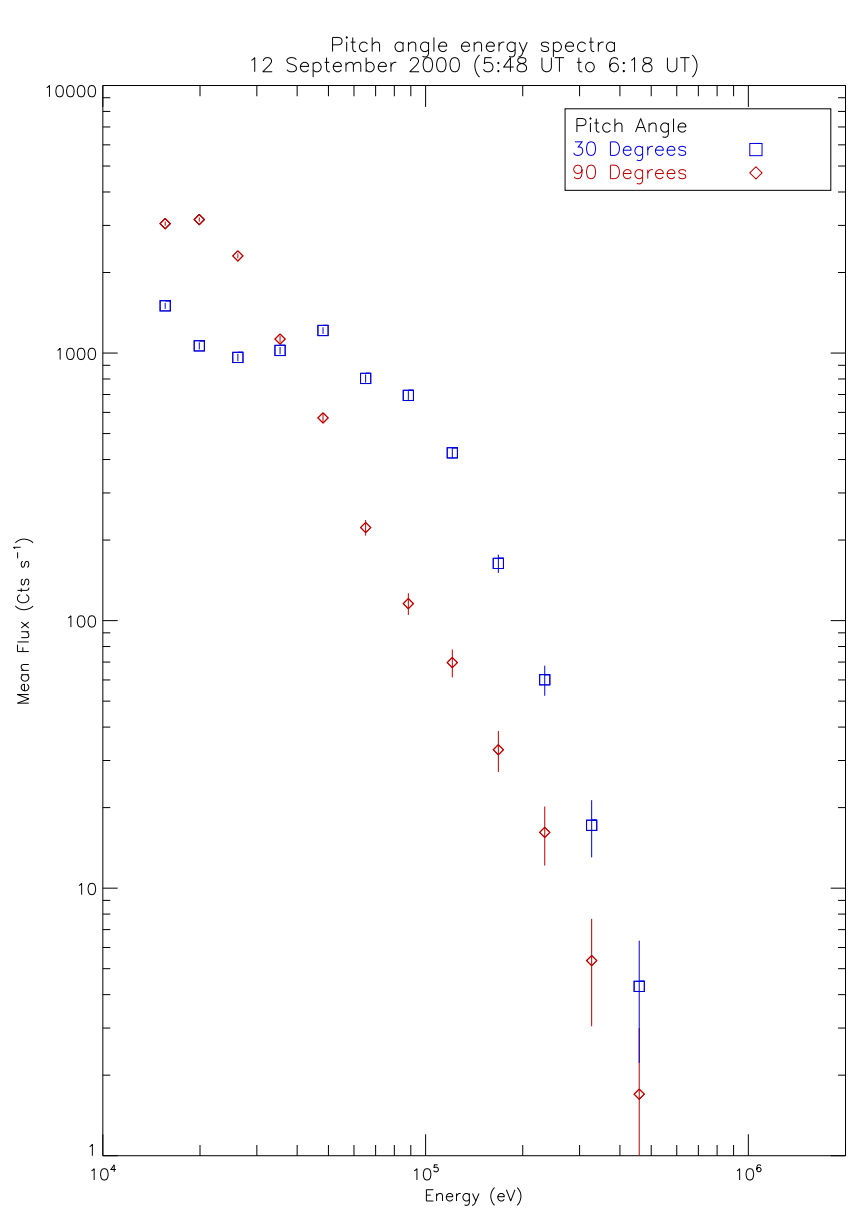

Fig. 12. Logarithmic plot of the mean flux of ions from 05:48 UT until 06:18 UT as a function of the ion energy. This half hour time period encompasses the butterfly PAD observed by Polar on 12 September $2000.30^{\circ}$ and $90^{\circ}$ pitch angles are shown to have unique energy spectra.

the nightside equatorial zone outside of $5.5 R_{E}$. Similarly, the onset time and duration of the butterfly PAD are not dependent on the energy for the two specific cases presented here.

The ions, however, demonstrate an energy dependent bifurcation in the observed PADs. Above energies of $\sim 60 \mathrm{keV}$, butterfly PADs exist in the ion population that are similar to those observed in the electrons. A higher energy level corresponds to an earlier detection of the onset of the butterfly PAD. For ions with energies below $\sim 60 \mathrm{keV}$, there appears to be a source centered upon a pitch angle of $90^{\circ}$. These source particles are on the same gradient drift paths as the electrons and higher energy ions, which have been modeled backward in time to show they contacted the magnetopause.

Where the magnetic field is still dipolar, which is usually inside a radial distance of $8 R_{E}$, the electric field has a decreasing impact on charged particles with higher energies. 
This allows for populations observed at a particular time and location with different energies to have varying origins.

Additionally, with the development of this model, it has been possible to determine the origin of energetic ions observed by the Polar satellite as functions of both pitch angle and location. The magnetopause can act simultaneously as a sink for high energy ions and electrons with pitch angles near $90^{\circ}$ via magnetopause shadowing, and as a source for ions with pitch angles near $90^{\circ}$ below $\sim 60 \mathrm{keV}$. This source population seems to be composed of a series of intensifications where the $90^{\circ}$ pitch angle ions are observed first followed progressively later by off equatorially mirroring particles.

Acknowledgements. This research has been supported by NASA grants NAG5-11397 and NNG05GD23G. The authors would also like to acknowledge J. H. King and N. Papatashvilli at Perot Sys, NASA GSFC and CDAWeb for providing solar wind data.

Topical Editor I. A. Daglis thanks P. Newell and another anonymous referee for their help in evaluating this paper.

\section{References}

Ashour-Abdalla, M., Bosqued, J. M., El-Alaoui, M., Peroomian, V., Zelenyi, L. M., Walker, R. J., and Wright, J.: A stochastic sea: The source of plasma sheet boundary layer ion structures observed by Cluster, J. Geophys. Res. (Space Physics), 110, 12221-12238, doi:10.1029/2005JA011183, 2005.

Blake, J. B., Fennell, J. F., Friesen, L. M., Johnson, B. M., Kolasinski, W. A., Mabry, D. J., Osborn, J. V., Penzin, S. H., Schnauss, E. R., Spence, H. E., Baker, D. N., Belian, R., Fritz, T. A., Ford, W., Laubscher, B., Stiglich, R., Baraze, R. A., Hilsenrath, M. F., Imhof, W. L., Kilner, J. R., Mobilia, J., Voss, D. H., Korth, A., Gull, M., Fisher, K., Grande, M., and Hall, D.: Ceppad, Space Sci. Rev., 71, 531-562, 1995.

Bogott, F. H. and Mozer, F. S.: Equitorial electron angular distributions in the loss cone and at large angles, J. Geophys. Res., 76, 6790-6805, 1971.

Fritz, T. A., Alothman, M., Bhattacharjya, J., Matthews, D. L., and Chen, J.: Butterfly pitch-angle distributions observed by ISEE-1, Planet. Space Sci., 51, 205-219, 2003.

Kaye, S. M., Lin, C. S., Parks, G. K., and Winckler, J. R.: Adiabatic modulation of equatorial pitch angle anisotropy, J. Geophys. Res., 83, 2675-2682, 1978.
Pfitzer, K. A., Lezniak, T. W., and Winckler, J. R.: Experimental verification of drift shell splitting in the distorted magnetosphere, J. Geophys. Res., 74, 4687-4693, 1969.

Richard, R. L., El-Alaoui, M., Ashour-Abdalla, M., and Walker, R. J.: Modeling the entry and trapping of solar energetic particles in the magnetosphere during the November 24-25, 2001 storm, J. Geophys. Res. (Space Physics), 114, 4210-4225, doi:10.1029/ 2007JA012823, 2009.

Roederer, J. G.: On the adiabatic motion of energetic particles in a model magnetosphere, J. Geophys. Res., 72, 981-992, 1967.

Roederer, J. G.: Dynamics of Geomagnetically Trapped Radiation, no. 2 in Physics and Chemistry in Space, Springer-Verlag, Berlin, 1970.

Russell, C. T., Snare, R. C., Means, J. D., Pierce, D., Dearborn, D., Larson, M., Barr, G., and Le, G.: The GGS/Polar Magnetic Fields Investigation, Space Sci. Rev., 71, 563-582, 1995.

Serlemitsos, P.: Low-energy electrons in the dark magnetosphere, J. Geophys. Res., 71, 61-77, 1966.

Stern, D. P.: Charged particle motions in a magnetic field that reduce to motions in a potential, Am. J. Phys., 43, 689-694, doi: 10.1119/1.9714, 1975.

Tsyganenko, N. A.: Modeling the Earth's magnetospheric magnetic field confined within a realistic magnetopause, J. Geophys. Res., 100, 5599-5612, 1995.

Tsyganenko, N. A. and Sitnov, M. I.: Modeling the dynamics of the inner magnetosphere during strong geomagnetic storms, J. Geophys. Res., 110, 3208-3223, doi:10.1029/2004JA010798, 2005.

Volland, H.: A model of the magnetospheric electric convection field, J. Geophys. Res., 83, 2695-2699, 1978.

Walker, R. J., Ashour-Abdalla, M., Ogino, T., Peroomian, V., and Richard, R. L.: Modeling Magnetospheric Plasma Sources and Losses, AGU Spring Meeting Abstracts, 2001.

West Jr., H. I.: Some observations of the trapped electrons produced by the Russian high-altitude nuclear detonation of October 28, 1962, in: ASSL Vol. 5: Radiation Trapped in the Earth's Magnetic Field, edited by: McCormac, B. M., pp. 634-662, 1966.

West Jr., H. I. and Buck, R. M.: Pitch angle distributions of energetic electrons in the equatorial regions of the outer magnetosphere - OGO-5 observations, in: Magnetospheric physics: proceedings of the Advanced Summer Institute held at Sheffield, UK, August 1973, edited by: McCormac, B. M., pp. 93-104, D. Reidel Publishing Co., Dordrecht, 1974.

West Jr., H. I., Buck, R. M., and Walton, J. R.: Electron pitch angle distributions of energetic electrons throughout the magnetosphere as observed on OGO 5, J. Geophys. Res., 78, 1064-1081, 1973. 